\title{
Valoración transversal tras quince años en una muestra de adictos a opiáceos en Asturias
}

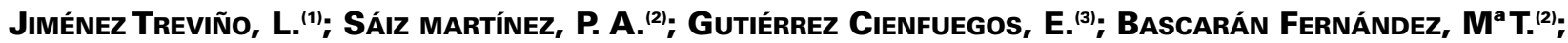 \\ Carreño Rendueles, e. ${ }^{(4)}$; González-Quiros Menéndez de luarca, M. ${ }^{(5)}$; \\ González García-Portilla, $\mathbf{M}^{\mathbf{a}} \mathbf{P}^{(2)}$; Bobes García, J.(2) \\ (1) Hospital Ramón y Cajal. Madrid. \\ (2) Área de Psiquiatría. Facultad de Medicina. Universidad de Oviedo. \\ (3) Servicios de Salud Mental del Principado de Asturias (SESPA). \\ (4) Clínica Médico Psicológica Asturias. Gijón. \\ (5) Clínica San Rafael.Oviedo.
}

\section{RESUMEN}

Se ha valorado la situación a los 15 años de una población de 215 pacientes adictos a opiáceos, todos los enrolados en Asturias durante los años 1980-83, en un programa de mantenimiento con metadona oral. Durante el período 1998-99 se procedió a la localización de los sujetos y recogida de los datos mediante un protocolo "ad hoc" y entrevistas telefónicas y/o revisión de historiales médicos. De la población inicial se ha podido localizar a un total de 74 pacientes, de los cuales el 35.1\% había fallecido, siendo la causa de muerte más importante el SIDA, seguido de la sobredosis. El 64.9\% restante permanecía vivo [edad media (SD) 38.7 (7.68); 82.4\% varones]. La mayoría (68.8\%) vivía en zonas urbanas y la convivencia fundamental era en pareja (52.1\%), estando casados un $45.8 \%$. El 41.9\% había sido detenido y el 29.3\% había estado en prisión en alguna ocasión. La mayoría eran politoxicómanos y la edad de inicio del consumo de heroína se situaba en los 18.4 años. El $41.7 \%$ era $\mathrm{VIH}+$, el $54.2 \% \mathrm{VHB}+\mathrm{y}$ el $41.7 \% \mathrm{VHC}+$. Se encontraron antecedentes familiares de adicción a drogas en el $50 \%$ de los sujetos, siendo la esposa/compañera la que más frecuentemente (45.8\%) presentaba alguna adicción. Conclusiones: esta población de heroinómanos que iniciaron mantenimiento con metadona hace más de 15 años, se caracteriza por una elevadísima mortalidad, importante deterioro legal y un notable nivel de discapacidad (especialmente en la función laboral, familiar y restricción en la participación social).

Palabras clave: heroinómanos, mantenimiento con metadona.

\section{SUMMARY}

A cross-sectional evaluation after 15 years was made in a total of 215 patients who had enrolled in a maintenance methadone program between 1980-1983. Data were collected (1998-1999) by means of an "ad hoc" protocol (sociodemographic, clinical, family, job and legal data). Information was collected by telephone interviews and/or revision of medical reports. Out of the initial population information was obtained on 74 were found. The death rate was $35.1 \%$. The major causes of death were AIDS and overdose. The mean age (SD) of those remaining alive was 38.7 (7.68); gender: $82.4 \%$ were males; residence: $68.8 \%$ lived in urban areas; civil status: $52.1 \%$ partners, $45.8 \%$ married; arrested: $41.9 \%$; previously imprisoned: $29.3 \%$. The majority were polydrug abusers. Age of initial consumption of heroin was 18.4 years. HIV+: $41.7 \%$, HBV+: $52.4 \%, \mathrm{HCV}$ : $41.7 \%$. Previous family history of addiction was found in $50 \%$ of the subjects, the wife or partner being the most frequent $(45.8 \%)$. Conclusions: this population is characterised by strikingly high mortality, high levels of disability (work, family and social participation) and infringements with the law.

Key words: heroin addicts, methadone maintenance program.

\section{INTRODUCCIÓN}

xisten insuficientes publicaciones de estudios de seguimiento a largo plazo de muestras de heroinómanos en nuestro país, lo cual implica una falta de información sobre un grupo poblacional de gran importancia, tanto por su número [la tasa de prevalencia de adicción a heroína en España se estima entre 2.4 y 17.6 por cada mil habitantes (1)] como por los problemas socio-sanitarios asociados con la adicción a opiáceos por vía parenteral [recordemos que constituye el mayor grupo de riesgo para la infección por VIH en nuestro país (2)]. 
Cuando se analizan los trabajos publicados en España hasta la fecha $(2,3,4,5,6)$ se aprecian carencias importantes entre las que destaca el tiempo de seguimiento. Proyectos ambiciosos como el Estudio Multicéntrico de Evaluación Y Seguimiento de Toxicómanos (EMETYST) (3) se limitan a dos años de seguimiento, lo que impide valorar el impacto de patologías crónicas como el SIDA o la influencia a largo plazo de las diferentes actuaciones sociosanitarias sobre este colectivo.

El objetivo de nuestro trabajo se centró en observar la evolución naturalística de un grupo de adictos a opiáceos a largo plazo, 15 años después del primer contacto, sin haber realizado, los investigadores, ningún tipo de actuación o control sobre la muestra durante todo este tiempo, con el fin de evitar posibles sesgos en el estado de salud de la cohorte. Por ello, se analizaron las condiciones sociodemográficas, toxicológicas, legales y clínicas con el fin de investigar tanto la morbi-mortalidad como la problemática social y médico-legal asociada al consumo de heroína, así como comprobar los efectos de la irrupción del virus del SIDA en este grupo poblacional.

\section{MATERIAL Y MÉTODOS}

Durante los años 1998 y 1999 se trató de evaluar una población compuesta por todos los pacientes registrados y autorizados por la Consejería de Sanidad del Principado de Asturias para integrarse en un Programa de Mantenimiento con Metadona (PMM) [215 pacientes (166 hombres y 49 mujeres) adictos a opiáceos que fueron registrados durante los años 19801983].

La información se obtuvo directamente mediante entrevista telefónica y/o a través de la revisión de historiales clínicos, considerándose como válidos los datos con fecha posterior al año 1995. Los sujetos fueron informados tanto de la confidencialidad de los datos como del conocimiento de la mayoría de sus antecedentes legales y toxicológicos con la finalidad de obtener mayor veracidad en las respuestas.

La evaluación se realizó a través de un protocolo "ad hoc" con un total de 38 ítems, en el que se incluyeron variables sociodemográficas, clínicas y legales, además de datos sobre la historia toxicológica personal y los antecedentes familiares. El análisis de los datos se llevó a cabo mediante el programa estadístico SPSS/PC+.

La validez de los datos recogidos se ha controlado mediante la comparación con los historiales médicos de los pacientes. El consumo actual de drogas o la abstinencia no ha sido comprobado mediante análisis de orina.

\section{RESULTADOS}

En esta evaluación se ha podido localizar a un total de 74 pacientes, lo que supone aproximadamente un tercio de la muestra inicial (34.4\%). De estos el $82.4 \%(n=61)$ son varones y el $17.6 \%(n=13)$ mujeres (la muestra inicial estaba compuesta por un $77.2 \%$ de hombres y un $22.8 \%$ de mujeres), y la media (SD) de edad de la muestra es de 38.7 (7.68) años.

De estos pacientes un 35.1\% ( $n=26)$ había fallecido en el momento del seguimiento (tabla 1). La principal causa de muerte fue el SIDA o patologías relacionadas con el SIDA con un $46 \%$ de las muertes, seguido de sobredosis con un $23 \%$, mientras que el $30 \%$ restante corresponde a accidentes y "otras causas" junto con las muertes de causa desconocida.

\section{Tabla 1. Análisis mortalidad 15 años}

\begin{tabular}{lrr}
\hline \% fallecidos & $35.1 \%$ & $(26)$ \\
\hline $\begin{array}{c}\text { \% fallecidos según sexo } \\
\text { Varones }\end{array}$ & $39.3 \%$ & $(24)$ \\
Mujeres & $15.4 \%$ & $(2)$ \\
\hline Causas fallecimientos & & \\
SIDA & $46.1 \%$ & $(12)$ \\
Sobredosis & $23.1 \%$ & $(6)$ \\
Accidente & $3.8 \%$ & $(1)$ \\
Otras/desconocido & $27 \%$ & $(7)$ \\
\hline Edad media (SD) & & \\
fallecimiento & 31.9 & $(4.2)$ años \\
\hline
\end{tabular}

Variables sociodemográficas y legales (tabla 2): de los pacientes localizados que estaban vivos ( $n=48)$, el $45.8 \%$ estaban casados y el $35.4 \%$ tenía hijos; la convivencia fundamental era en pareja $(52.1 \%)$ seguida de la convivencia con los padres (18.8\%), y el $81.3 \%$ tenía una residencia estable. El nivel de escolarización en la mayor parte de los casos consistía en estudios primarios $(39.6 \%)$ con un pequeño porcentaje que había cursado estudios superiores (4.2\%). Un $27.1 \%$ tenía trabajo fijo, siendo la mayoría obreros no cualificados $(28.4 \%)$, y el $31.3 \%$ señalaba tener unos ingresos suficientes.

La mayor parte de la muestra pertenecía a zonas urbanas $(68.8 \%)$, siendo la mayoría de Oviedo (37.5\%) seguido de Mieres (18.8\%), Gijón (8.3\%) y Avilés $(4.2 \%)$.

El $41.9 \%$ de la muestra (20 sujetos) había sido detenido en alguna ocasión, con una media (SD) de 4.8 (4.54) detenciones, mientras que un 18.8\% nunca había sido detenido. Un $29.3 \%$ de sujetos había estado alguna vez en prisión, con una media (SD) de 2.5 (1.91) encarcelamientos y una estancia media (SD) en la cárcel de 19 (19.1) meses. En el momento de la 
Tabla 2. Características sociodemográficas de los sujetos vivos ( $n=48$ )

\begin{tabular}{|c|c|}
\hline Sexo & $\begin{array}{l}\text { Varones } 77.1 \%(37) \\
\text { Mujeres } 22.9 \%(11) \\
\end{array}$ \\
\hline Edad media (SD) & 38.7 (7.68) años \\
\hline Estado civil & $\begin{array}{l}\text { Casado/a } 45.8 \%(22) \\
\text { Soltero/a } 20.8 \%(10) \\
\text { Separado-divorciado/a 12.6\% (6) } \\
\text { Viudo/a 6.3\% (3) } \\
\text { Desconocido } 14.5 \%(7)\end{array}$ \\
\hline Hijos & $\begin{array}{l}\text { Sí 35.4\% (17) } \\
\text { No 37.5\% (18) } \\
\text { Desconocido 27.1\% (13) }\end{array}$ \\
\hline Convivencia & $\begin{array}{l}\text { Esposa-compañera/o 52.1\% (25) } \\
\text { Padres } 18.8 \%(9) \\
\text { Pensión o similar } 2.1 \%(1) \\
\text { Desconocido } 27.0 \%(13)\end{array}$ \\
\hline Lugar de residencia & $\begin{array}{l}\text { Urbano 68.8\% (33) } \\
\text { Rural 27.0\% (13) } \\
\text { Desconocido 4.2\% (2) }\end{array}$ \\
\hline Escolarización & $\begin{array}{l}\text { Estudios primarios } 39.6 \%(19) \\
\text { Estudios medios } 27.1 \%(13) \\
\text { Estudios superiores } 4.2 \%(2) \\
\text { Desconocido } 29.1 \%(14)\end{array}$ \\
\hline Profesión & $\begin{array}{l}\text { Obrero no cualificado 25.0\% (12) } \\
\text { Obrero semi-cualificado 16.7\% (8) } \\
\text { Oficinista / téc. medio 16.7\% (8) } \\
\text { Téc. superior/prof.liberal. 4.2\% (2) } \\
\text { Otros } 4.2 \%(2) \\
\text { Desconocido 23.2\% (16) }\end{array}$ \\
\hline Situación laboral & $\begin{array}{l}\text { Fijo 27.1\% (13) } \\
\text { Eventual 6.3\% (3) } \\
\text { Paro con subsidio 6.3\% (3) } \\
\text { Paro sin subsidio 16.7\% (8) } \\
\text { Jubilado } 10.4 \% \text { (5) } \\
\text { Incapacidad laboral 8.4\% (4) } \\
\text { Minusvalía 4.2\% (2) } \\
\text { Estudiante 2.1\% (1) } \\
\text { Desconocido 18.5\% (9) }\end{array}$ \\
\hline $\begin{array}{l}\text { Situación económica } \\
\text { actual }\end{array}$ & $\begin{array}{l}\text { Ingresos suficientes 31.3\% (15) } \\
\text { Ingresos esporádicos } 4.2 \% \text { (2) } \\
\text { Depende de su familia } 12.5 \% \text { (6) } \\
\text { Depende de otras pers. } 2.1 \% \text { (1) } \\
\text { Desconocido } 49.9 \%(24)\end{array}$ \\
\hline $\begin{array}{l}\% \text { det. alguna ocasión } \\
\text { media (SD) } n^{\circ} \text { detenc. }\end{array}$ & $\begin{array}{l}41.9 \%(20) \\
4.8(4,5)\end{array}$ \\
\hline $\begin{array}{l}\text { \% prisión alguna ocasión } \\
\text { med. (SD) veces en prisión } \\
\text { est. media (SD) en prisión }\end{array}$ & $\begin{array}{l}29.3 \%(14) \\
2.5(1.9) \\
19.0(19.1) \text { meses }\end{array}$ \\
\hline
\end{tabular}

encuesta sólo un $2.1 \%$ de la muestra tenía causas pendientes. En las variables legales no se dispone de datos de 19 sujetos (39.4\% de la muestra).

Variables toxicológicas (tabla 3): la edad de inicio (años) del consumo de las diferentes drogas estudiadas fue (en orden ascendente): tabaco 13.4 (2.3), alcohol 15.7 (2.9), cannabis 15.8 (2.7), anfetaminas 16.7 (2.9), LSD 17.8 (1.9), heroína 18.4 (2.8), cocaína 19.6 (5.9), benzodiacepinas 20.2 (4.1), codeína 21.3 (3.7), metadona 23.7 (6.7), éxtasis 30 (7.1). Por otra parte, la droga consumida durante más tiempo [media (SD) de años de consumo] sería el tabaco [22.1 (6.9)], seguido de alcohol [18.0 (8.2)] y heroína [15.4 (5.5)]. De las personas que respondieron a la pregunta sobre el tipo de droga preferida el $96.9 \%$ eligió a la heroína, seguida del cannabis $(3.1 \%)$.

\section{Tabla 3. Politoxicomanía: edad de inicio y años} de consumo medios (SD)

\begin{tabular}{lcc}
\hline & $\begin{array}{c}\text { Edad de } \\
\text { inicio }\end{array}$ & $\begin{array}{c}\text { Años de } \\
\text { consumo }\end{array}$ \\
\hline Tabaco & $13.4(2.3)$ & $22.1(6.9)$ \\
Alcohol & $15.7(2.9)$ & $18.0(8.2)$ \\
Cannabis & $15.8(2.7)$ & $13.5(8.5)$ \\
LSD & $17.8(1.9)$ & $2.1(2.2)$ \\
Heroína & $18.4(2.8)$ & $15.4(5.5)$ \\
Cocaína & $19.6(5.9)$ & $8.7(7.8)$ \\
Benzodiacepinas & $20.2(4.1)$ & $9.8(7.3)$ \\
Codeína & $21.3(3.7)$ & $3.4(3.3)$ \\
Metadona & $23.7(6.7)$ & $5.1(3.7)$ \\
Éxtasis & $30.0(7.1)$ & $0.7(0.6)$ \\
$n=48$ & & \\
\end{tabular}

Sólo se consiguieron datos de 20 de los pacientes sobre el consumo actual (no comprobado mediante análisis de orina) con un $45 \%$ de consumidores frente a un 55\% de abstinentes.

Recursos sanitarios utilizados: aparte del PMM (1980-1983), que fue el criterio de inclusión exigido para este estudio de seguimiento, los diferentes recursos asistenciales han sido frecuentados en la siguiente proporción: desintoxicación ambulatoria $46 \%$, desintoxicación hospitalaria 33.4\%, programa de mantenimiento con naltrexona (PMN) 27.1\%, comunidad terapéutica $18.8 \%$, centro de día $4.2 \%$. Las medias (SD) de abstinencias conseguidas en los diferentes tratamientos fueron: desintoxicación ambulatoria 32.4 (36.4) meses, desintoxicación hospitalaria 6 (8.3) meses, comunidad terapéutica 19.6 (13.3) meses, centro de día 1 mes, PMN 42.3 (67.3) meses, PMM 39.5 (25.3) meses. Actualmente el $4.2 \%$ no sigue ningún tratamiento, el $4.2 \%$ desintoxicación ambulatoria, el 25\% PMM, el 2.1\% PMN, y del 64.5\% restante no se tienen datos. 
Variables clínicas (tabla 4): de los pacientes vivos ( $n=48)$, un $54.2 \%$ tenía anticuerpos positivos para VHB, el $43.8 \%$ para VHC, y el $2.1 \%$ estaba diagnosticado de cirrosis. Un $41.7 \%$ era VIH + y el $27.2 \%$ había presentado patologías relacionadas con el SIDA (candidiasis, tuberculosis, herpes, gonorrea). Del resto de patologías destacan las de tipo digestivo (10.4\% de la muestra), traumatológico (6.3\%) y psiquiátrico (4.2\%).

\section{Tabla 4. Variables clínicas}

\section{$\mathrm{VIH}+$}

Infecciones secundarias*

Virus Hepatitis B+

Virus hepatitis $\mathrm{C}_{+}$

Cirrosis hepática

* herpes, lues, gonorrea, candidiasis,

tuberculosis, otras.

$n=48$

Antecedentes familiares (tabla 5): un 50\% de los pacientes vivos de la muestra tenía antecedentes familiares de adicción a drogas, y de estos un 25\% tenía antecedentes en más de un familiar. De los familiares que también presentaban alguna adicción, la esposa/o-compañera/o lo tenía en un $45.8 \%$ de los casos, los hermanos/as en un $29.2 \%$ y los padres en un $16.6 \%$.

\section{Tabla 5. Antecedentes familiares de enfermedad mental}

\begin{tabular}{lr}
\hline Adicción a drogas & $50.0 \%(24)$ \\
Esposa/o-compañera/o & $45.8 \%(11)$ \\
Hermano/a & $29.2 \%(7)$ \\
Padre/madre & $16.6 \%(4)$ \\
Hijo/a & $4.2 \%(1)$ \\
Abuelo/a & $4.2 \%(1)$ \\
\hline Psicopatología & $33.4 \%(16)$ \\
Esquizofrenia & $2.1 \%(1)$ \\
Trastornos Neuróticos & $8.4 \%(4)$ \\
Trastornos Afectivos & $8.4 \%(4)$ \\
Trastornos de la Personalidad & $10.5 \%(5)$ \\
Trastorno Mental Orgánico & $4.2 \%(2)$ \\
\hline $\mathrm{n}=48$ & \\
\hline
\end{tabular}

En cuanto a los antecedentes familiares de patología psiquiátrica un $2.1 \%$ tenía antecedentes de esquizofrenia, un $8.4 \%$ antecedentes de trastorno del humor, un $4.2 \%$ antecedentes de trastorno mental orgánico, un $8.4 \%$ de trastornos de tipo neurótico y u $10.5 \%$ tenía antecedentes de trastornos de personalidad en su familia.

La mayoría de los entrevistados señalaba tener una buena convivencia $(37.5 \%)$ con la familia, calificando los cambios en la vida familiar como moderados o ninguno; sólo un $10.4 \%$ señaló cambios marcados en el modo de vida familiar.

\section{DISCUSIÓN}

Varios autores han señalado la dificultad que supone hacer estudios de seguimiento en adictos a drogas $(6,7,8)$ ya que constituyen un grupo de población marginal, poco motivada y colaboradora, con gran tendencia a la emigración y con problemas legales asociados en gran parte de los casos, lo cual implica que estudios de la importancia del proyecto EMETYST (3) sólo han podido localizar en torno al $60 \%$ de la muestra tras dos años de seguimiento. En este sentido, consideramos importante haber podido localizar a un tercio de la muestra tras 15 años de evolución libre. Además, el hecho de no haber sometido a los pacientes a ningún tipo de control, elimina el sesgo en cuanto al mejor estado de salud que puede tener una cohorte controlada periódicamente.

El primer dato a favor de la validez de los resultados obtenidos es el porcentaje de fallecidos observado en la muestra (38.5\%) que es similar al consignado en otros seguimientos a largo plazo como el de Vaillant (9) en Estados Unidos (con una mortalidad en torno al $2 \%$ anual), Oppenheimer y cols. (10) en Londres (1.8\% anual), o los estudios escandinavos de Haastrup y Jepsen (11) (2.4\% anual) y Engstrom y cols. (12) (3.1\% anual).

Nuestro trabajo difiere de la mayoría de los estudios $(10,11,12,13)$ que señalan a la sobredosis como causa principal de muerte entre los heroinómanos, ya que casi la mitad de los fallecimientos de nuestra cohorte $(46 \%)$ ha estado relacionada con el SIDA. Esta discordancia puede ser debida a varios factores: por un lado está el tiempo de seguimiento, que en muchos de los estudios es inferior a dos años y, en consecuencia, es difícil que se pueda reflejar la incidencia de una enfermedad de carácter crónico como es el SIDA. Por otra parte, como ya apuntan Oppenheimer y cols. (10) en su estudio, muchas de las cohortes de los grandes estudios a largo plazo pertenecen a una época (años 70) en la que el virus del SIDA todavía no había hecho acto de presencia entre estos colectivos.

Varios autores sugieren una menor mortalidad entre las mujeres adictas a heroína comparadas con los hombres $(10,14,15,16,17,18)$. En este sentido, aunque en nuestro estudio se confirma esa menor mortalidad (15.4\% las mujeres y $39.3 \%$ los hombres), no se obtienen diferencias estadísticamente significativas.

Por último cabe reseñar, que los datos de mortalidad obtenidos en este estudio son muy superiores a 
los datos obtenidos en nuestra región para el grupo de edad de 30-34 años [recuérdese que la edad media de los fallecidos fue de 31.9 (4.2) años]. Así, el porcentaje de fallecidos de ambos sexos, para todas las causas, sería en Asturias (19), en el año 1995, de 0.14\% (0.22\% para los varones y $0.61 \%$ para las mujeres) frente al $35.1 \%$ (39.3\% para los varones y $15.4 \%$ para las mujeres) constatado en nuestra muestra. De igual modo, las muertes por SIDA constituyen el 30.8\% de los fallecimientos en nuestra región en ese grupo de edad (20), frente al $46.1 \%$ hallado en nuestra muestra, mientras que las muertes por accidente de tráfico supondrían un $5.1 \%$ (20) frente al 3.8\%.

Lo constatado anteriormente subraya, por una parte, la elevadísima mortalidad que se constata en muestras de adictos a opiáceos cuando son comparadas con sus coetáneos de población general y, por otra, la gran trascendencia que el SIDA tiene, como causa de muerte, en aquellos pacientes que iniciaron su toxicomanía en torno a los años 80.

Variables sociodemográficas y legales: al igual que en la mayoría de estudios, tanto españoles $(2,3,4,5$, $6,21)$ como internacionales $(9,10,11,12,13,22)$, la muestra estaba compuesta mayoritariamente por varones $(82.4 \%)$, con predominio del lugar de residencia en el medio urbano y de estado civil soltero.

El nivel de escolarización de la muestra estudiada es, en líneas generales, superior al hallado en el Padrón Municipal de Asturias de 1996 (23), según el cual el $19 \%$ de mayores de 10 años tienen estudios primarios, el $19 \%$ estudios secundarios y el $9 \%$ ha cursado estudios superiores. Si bien, hay que tener en cuenta que dichos porcentajes están referidos a todos los grupos de edad, con lo que son, a todas luces, inferiores a los que se esperaría encontrar en grupos de edad de 35-39 años, que es dónde se encuadra nuestra muestra. Por otra parte, y a pesar de ello, el porcentaje de personas con estudios superiores es inferior en nuestra muestra (4.2\%), lo cual pone de manifiesto la existencia de un menor porcentaje de personas con estudios superiores entre las muestras de adictos a opiáceos que entre población general.

En cuanto a la situación laboral destacar que un $27.1 \%$ de la muestra tenía un trabajo fijo lo cual contrasta de modo llamativo con las tasas de actividad registradas durante el año 1998 en nuestra región (24) para los grupos de edad de 25-54 años, que se sitúan en torno al 68.6, e indican la gran problemática laboral de este colectivo.

El Informe anual de la Unión Europea sobre el problema de las drogas (1) incide en la importancia de los delitos relacionados con el uso de las drogas, bien sea con la intención de obtener dinero para su adquisición, bien los cometidos bajo la influencia de las drogas, bien otros delitos relacionados con el mundo de la drogadicción como el crimen organizado, la prostitu- ción o el propio tráfico de drogas (1). Los resultados de nuestro estudio corroboran la magnitud de la problemática legal en este colectvo con cifras de detenciones y estancia en prisión superiores a otros trabajos llevados a cabo en nuestro país $(3,6)$, probablemente por el mayor tiempo de evolución de nuestra cohorte.

Variables toxicológicas: coincidiendo con otros estudios $(3,5)$ la mayoría de los heroinómanos eran politoxicómanos y comenzaron a consumir heroína en torno a los 18 años. Si se analizan las edades de inicio en las diferentes drogas se puede establecer un patrón de consumo que se repetiría en la mayoría de los politoxicómanos. Las primeras drogas que se consumen serían las drogas legales (tabaco y alcohol), en la adolescencia, constituyendo el cannabis la "puerta de entrada" a las sustancias ilegales; posteriormente se usarían las drogas alucinógenas y, sobre los 18-20 años, se iniciaría el consumo de las drogas más dependígenas como heroína y cocaína. Una última fase comprendería el uso de metadona y otros opiáceos como sustitutivos de la heroína. Hay que resaltar que el inicio del consumo de éxtasis en nuestra cohorte es muy tardío (30 años) debido a que el uso de esta droga no se popularizaría hasta finales de los años ochenta (25).

Al confrontar edades de inicio y años de consumo de las diferentes drogas se puede comprobar que el consumo de tabaco, alcohol y heroína se mantiene constante desde el inicio de la adicción a cada droga, mientras que el resto de sustancias se consume de una manera más irregular en el tiempo, lo que concuerda con la masiva respuesta a favor de la heroína en la pregunta sobre el tipo de droga preferida.

A pesar de haber obtenido un índice de respuesta muy bajo a la pregunta sobre el consumo actual (27\%), los datos obtenidos (54\% de abstinentes) concuerdan con otros estudios españoles $(3,5)$ e internacionales $(9,10)$. Una de las reglas postuladas por Vaillant (26) sobre las adicciones a drogas en la que afirma que la abstinencia en una cohorte de adictos a heroína de larga evolución se produce, aproximadamente, en un 3\% cada año, se confirma también en nuestro estudio de seguimiento. Haastrup y Jepsen (11) corroboran esta teoría en su seguimiento a 11 años de 300 adictos a opiáceos de Copenhague y afirman que la posibilidad de conseguir la abstinencia se mantiene constante en el tiempo.

Uso de recursos sanitarios: la norma es la utilización de los diferentes dispositivos asistenciales según se han ido ofreciendo, con medias del número de veces de utilización de cada posibilidad terapéutica superiores a 1 en la práctica totalidad de los casos. El PMM es el más frecuentado [media (SD) 2.3 (1.3)] ya que la muestra estaba compuesta por adictos a opiáceos que acudieron a este tipo de programas en, al menos, una ocasión. El mayor tiempo de abstinencia 
medio, en cambio, se conseguía en los PMN. Esto puede estar ligado a los criterios de inclusión/exclusión utilizados habitualmente en este tipo de programas en los que participan los sujetos más motivados para el tratamiento, con mayor educación, mejor ajuste psicológico, menor historial toxicológico y menor tasa de criminalidad (27).

Variables clínicas: los datos de nuestro estudio sobre la seroprevalencia del $\mathrm{VIH}(41.7 \%$ de los pacientes localizados vivos y $48.6 \%$ si tenemos en cuenta los datos de los pacientes ya fallecidos) concuerdan con los publicados en otros estudios $(2,5,28)$, incluido el de Delgado-Iribarren y cols. (2) sobre la infección por VIH en adictos a drogas por vía parenteral, y confirman la magnitud del problema en este colectivo (recordemos que el SIDA ha resultado ser la causa principal de mortalidad en nuestro estudio).

De la misma manera, se confirma la alta prevalencia de anticuerpos frente a los virus hepatótrofos, ya observada en otros trabajos $(2,4,5,21,28)$, y que es producto, al igual que la transmisión del VIH, de la inyección por vía parenteral de la droga y del hábito, todavía arraigado entre nuestros heroinómanos, de compartir las jeringuillas.

Antecedentes familiares: en la muestra se constata una alta prevalencia de antecedentes familiares de consumo de drogas, sobre todo en esposas/os-compañeras/os, y antecedentes de psicopatología. Aunque no está recogido en los resultados, en la mayoría de los casos la adicción de los padres era a alcohol mientras que los hermanos/as y esposas/os-compañeras/os habitualmente consumían el mismo tipo de droga que el entrevistado.

\section{CONCLUSIONES}

1. En esta población de heroinómanos que iniciaron mantenimiento con metadona hace más de 15 años se constata una elevadísimo porcentaje de fallecimientos.

2. Resulta imprescindible fomentar las estrategias encaminadas a prevenir y a tratar las patologías infecto-contagiosas y sus complicaciones, así como llevar un buen control serológico de estos pacientes e incidir en los programas de reducción del daño.

3. La población estudiada se caracteriza por un importante deterioro legal y un notable nivel de discapacidad, especialmente en la función laboral, familiar y restricción en la participación social.

4. Por último, se observa, en esta población, una elevada prevalencia de antecedentes familiares de trastornos mentales y del comportamiento, que confirma la necesidad de atención a la salud mental de la familia y del paciente.

\section{BIBLIOGRAFIA}

(1) EMCDDA. Annual Report on the State of the Drugs Problem in the European Union - 1997. Lisboa. European Monitoring Centre for Drugs and Drug Addiction. 1997.

(2) Delgado-Iribarren A, Wilhelmi I, Padilla B, Cañedo T, Gómez J, Elviro J. Infección por VIH y por los virus de la hepatitis B, C, y D en adictos a drogas por vía parenteral. Seroprevalencia de un año y su seguimiento. Enf Infec y Microbiol Clin. 1993; 11 (1): 813.

(3) Sánchez-Carbonell J, Brigos B, Camí J. Evolución de una muestra de heroinómanos dos años después del inicio del tratamiento (proyecto EMETYST). Med Clin (Barc). 1989; 135-139.

(4) Bobes J, Bousoño M, Fernández JM, Hinojal R. Evaluación prospectiva de 473 toxicodependientes en Asturias (1979-1982). Actas Luso-Esp Neurol Psiquiatr. 1984; 12 (5): 347-356.

(5) Marina PA. Adictos a la heroína en Asturias. Un estudio de seguimiento. En: Bobes J, GonzálezQuirós P, Portilla PG. 30 años de alcohol y otras drogas en Asturias. Oviedo. Gofer. 1996. 31-43.

(6) García A, Ezquiaga E. Estudio de seguimiento a seis meses de una muestra de drogodependientes. Arch de Neurobiol. 1992; 55 (5): 215-223.

(7) Walton MA, Ramanthan CS, Reischl TM. Tracking substance abusers in longitudinal research: understanding follow-up contact difficulty. Am J Community Psychol. 1998; 26 (2): 233-253.

(8) Cottler LB, Compton WM, Ben-Abdallah A, Horne M, Claverie D. Achieving a 96.6 percent follow-up rate in a longitudinal study of drug abusers. Drug Alcohol Depend. 1996; 41 (3): 209-217.

(9) Vaillant GE. A 20 year follow-up of New York narcotic addicts. Arch Gen Psychiatry. 1973; 29: 237-241.

(10) Oppenheimer E, Tobutt C, Taylor C, Andrew T. Death and survival in a cohort of heroin addicts from London clinics: a 22-year follow-up study. Addiction. 1994; 89: 1299-1308.

(11) Haastrup S, Jepsen PW. Eleven year follow-up of 300 young opioid addicts. Acta Psychiatr Scand, 1988; 77: 22-26.

(12) Engstrom A, Adamsson C, Allbeck P, Rydberg U. Mortality in patients with substance abuse: A follow-up in Stockholm County, 1973-1984. Int J Addict. 1991; 26: 91-106.

(13) Yih-Ing H, Douglas M, Powers K. A 24-year followup of California narcotic addicts. Arch Gen Psychiatry. 1993; 50: 577-584. 
(14) Colerige J, Cameron P, Drummer O, McNeil John. Survey of drug-related deaths in Vitoria. The Medical Journal of Australia, 1992; 157: 459-462.

(15) Hammersley R, Cassidy M, Oliver J. Drugs associated with drug-related deaths in Edinburgh and Glasgow. Addiction, 1995; 90: 959-965.

(16) De la Fuente L, Barrio L, Vicente J, Brajo MJ, Santacreu J. The impact of drug-related deaths on mortality among young adults in Madrid. Am J Public Health, 1995; 85: 102-105.

(17) Torralba L, Brugal MT, Villalbi JR, Tortosa MT, Toribio A, Valverde JL. Mortality due to acute adverse drug reactions: opiates and cocaine in Barcelona, 1989-1993. Addiction, 1996; 91: 419426.

(18) Pereiro C. Muerte tras reacción aguda por consumo de drogas en Galicia. Tesis Doctoral. Universidad de Santiago de Compostela. 1998.

(19) Consejería de Servicios Sociales. Informes técnicos. Mortalidad en Asturias 1995. Principado de Asturias. Consejería de Servicios Sociales. 1997.

(20) SADEl. Movimiento natural de la población de Asturias 1995. Principado de Asturias. Consejería de Economía. 1996.

(21) Urtiaga M, Extramiana E, Moreno C, Ardanaz E, Martínez M. Aproximación a la prevalencia de usuarios de Heroína en Navarra en 1990. Gac Sanit. 1993; 7: 56-62.

(22) Tobutt C, Oppenheimer E, Laranjeira R. Health of cohort of heroin addicts from London clinics: a 22 year follow up. BMJ. 1996; 312: 1458.

(23) SADEI. Datos y cifras de la economía asturiana 1996. Principado de Asturias. Consejería de Economía. 1997.

(24) SADEI. Estadísticas laborales 1998. Principado de Asturias. Gobierno del Principado de Asturias. 1999.

(25) Bobes J, Lorenzo P, Sáiz PA. Éxtasis (MDMA): Un abordaje comprehensivo. Barcelona. Masson. 1997.

(26) Vaillant GE. Outcome research in narcotic addiction - problems and perspectives. Am J Drug Alcohol Abuse. 1974; 1:25-36.

(27) Álvarez CE, Carreño JE, López JL, Ortiz R. Tratamientos con naltrexona. En: Bobes J, GonzálezQuirós P, Portilla PG. 30 años de alcohol y otras drogas en Asturias. Oviedo. Gofer. 1996. 135-146.

(28) Mandelli C, Cesana M, Ferroni P, Lorini GP, Aimo GP, Tagger A, Bianchi PA, Conte D. HBV, HDV, and HIV infections in 242 drug addicts: two-year followup. Eur J Epidemiol. 1988; 4 (3): 318-321. 
